\title{
Microwave-assisted extraction of chitosan from Rhizopus oryzae NRRL 1526 biomass
}

\author{
Joseph Sebastian $^{\text {a }}$, Tarek Rouissi ${ }^{\text {a, Satinder Kaur Brar }}{ }^{\text {a, b, }}{ }^{*}$, Krishnamoorthy Hegde $^{\text {a }}$, Mausam Verma ${ }^{\mathrm{c}}$ \\ a INRS-ETE, Université du Québec, 490, Rue de la Couronne, Québec, G1K 9A9, Canada \\ b Department of Civil Engineering, Lassonde School of Engineering, York University, North York, Toronto, Ontario, M3J 1P3, Canada \\ ' Institut de recherche et de développement en agroenvironnement (IRDA), 2700 Rue Einstein, Québec, QC G1P 3W8, Canada
}

\section{ART ICLE INFO}

\section{Keywords:}

Chitosan

Microwave

Extraction

Fungal biomass

R. oryzae

Degree of deacetylation

\begin{abstract}
A B S T R A C T
Microwave-assisted extraction (MAE) of chitosan from dried fungal biomass of Rhizopus oryzae NRRL1526, obtained by culturing on potato dextrose broth (PDB), was performed and the optimal conditions required were identified using statistical analysis for the first time in this study. This microwave-assisted extraction (MAE) was compared against the conventional autoclave assisted method of chitosan extraction. The full factorial experimental design was used to investigate the impact of operating parameters of MAE, microwave power (100 W-500 W), and duration (10 min-30 min), on alkaline insoluble material (AIM) yield, chitosan yield, and degree of deacetylation (DDA). The effect of operating conditions was then evaluated using full factorial data analysis and optimum condition for MAE of chitosan was identified using response surface methodology to be $300 \mathrm{~W}$ and $22 \mathrm{~min}$. This optimum condition identified was then further evaluated and the chitosan obtained characterized. Higher chitosan yield of $13.43 \pm 0.3 \%(\mathrm{w} / \mathrm{w})$ of fungal biomass was obtained when compared to that obtained, $6.67 \% \pm 0.3 \%(\mathrm{w} / \mathrm{w})$ of dry biomass, for the conventional extraction process. MAE yielded chitosan of higher degree of deacetylation, $94.6 \pm 0.9 \%$ against $90.6 \pm 0.5 \%$ (conventional heating), but the molecular weight was observed to be similar to that obtained by using conventional autoclave heating. MAE of chitosan was observed to yield a higher quantity of chitosan when compared to conventional extraction process and obtained chitosan exhibited a higher degree of deacetylation as well as molecular weight. The lower energy consumption of $0.11 \mathrm{kWh}$ for MAE ( $5 \mathrm{kWh}$ for conventional process) and the concomitant reduction in the energy bill to 1.1 cents from 50 cents, in addition to the above results, show that microwave irradiation is a more efficient and environment-friendly means to obtain chitosan from fungal biomass.
\end{abstract}

\section{Introduction}

Chitosan is the only pseudo-natural cationic polymer, made up of $\mathrm{N}$-glucosamine moieties, derived from chitin that is widely distributed in nature in organisms ranging from yeast to marine organisms, such as crustaceans (Rinaudo, 2006). The deacetylated form of chitin (considered to be the second most abundant polysaccharide after cellulose) possesses important properties, such as versatile biological activity, excellent biocompatibility, complete biodegradability, and low toxicity. These unique properties of the biopolymer have fueled its demand for applications in diverse fields such as wastewater treatment, pharmaceuticals, food and beverage, cosmetics and agricultural. Moreover, it has been awarded GRAS (Generally Regarded as Safe) status by the United State Food and Drug Association (US FDA) (Knorr \& Klein,
1986; Mourya \& Inamdar, 2008; Rajeshkumar et al., 2009; Rinaudo, 2006).

The current production of chitosan from chitin present in shellfish waste, which can take up to 2 days, involves stages of demineralization, deproteinization, and decolorization, using large quantities of toxic chemicals, prior to alkaline deacetylation of chitin to chitosan. The chitosan so obtained show inconsistencies in both physical and chemical properties, such as protein contamination, degree of deacetylation and variations rendered due to seasonal variations, which limits its application (Amorim, Souza, Fukushima, \& Campos-Takaki, 2001; Chatterjee, Adhya, Guha, \& Chatterjee, 2005; Knorr \& Klein, 1986; Kumari \& Rath, 2014). This dependence on crustaceans causes environmental pollution due to the generation of large quantities of highly alkaline and acidic effluents rich in proteinaceous waste materials. These limitations have necessitated the need for alternate means of chitosan production by the switch to fungal chitosan.

\footnotetext{
* Corresponding author at: INRS-ETE, Université du Québec, 490, Rue de la Couronne, Québec, G1K 9A9, Canada.

Email address: Satinder.Brar@lassonde.yorku.ca, satinder.brar@ete.inrs.ca (S.K. Brar)
} 
Chitin and chitosan are an integral part of the fungal cell wall of certain fungi especially those belonging to the class Zygomycetes (Nwe \& Stevens, 2004). Several yeasts and fungal species, such as Candida albicans, Saccharomyces cerevisiae, Mucor rouxii, Cunninghamella elegans, Gongronella butleri, Phycomyces blakesleeanus, Rhizopus spp., and Absidia spp., have been previously investigated for chitosan production (Amorim et al., 2001; Chatterjee et al., 2005; Chatterjee, Chatterjee, Chatterjee, \& Guha, 2008; Knorr \& Klein, 1986; Muzzarelli, Ilari, Tarsi, Dubini, \& Xia, 1994; Streit, Koch, Laranjeira, \& Ninow, 2009; Suntornsuk, Pochanavanich, \& Suntornsuk, 2002; White, Farina, \& Fulton, 1979; Yokoi et al., 1998). The chitosan obtained from fungal strains shows better uniformity in molecular weight, low polydispersity index and degree of deacetylation (Brown, Brunt, \& Rehmann, 2016; Suntornsuk et al., 2002). Moreover, extraction of chitosan from fungal sources require less amount of chemicals as only mild conditions are needed for extraction and do not require stages, such as demineralization and depigmentation. Hence, producing a lesser amount of toxic waste can be considered as a greener alternative (Dhillon, Kaur, Brar, \& Verma, 2012). The main limitation of the fungal chitosan production is low biomass production. Hence, an efficient extraction technique is required to obtain maximum chitosan yield from the available biomass.

Conventional alkaline extraction of chitosan is performed at high temperature and hence, consume a lot of energy and requires a lot of time. To this effect, the adoption of microwave heating in place of conventional heating can reduce the chitosan extraction time from hours to minutes to attain the same degree of deacetylation as traditional methods (Alishahi et al., 2011; El Knidri, El Khalfaouy, Laajeb, Addaou, \& Lahsini, 2016; Mahdy Samar, El-Kalyoubi, Khalaf, \& Abd El-Razik, 2013; Sagheer, Al-Sughayer, Muslim, \& Elsabee, 2009). The use of microwave irradiation is a more efficient and environment-friendly technique to extract animal chitosan as observed by El Knidri et al. (2016) and Mahdy Samar et al. (2013). During conventional heating, the reactants are slowly activated, not uniformly, whereas microwave heating happens at molecular level leading to a uniform rapid rise in temperature (El Knidri et al., 2016; Mahdy Samar et al., 2013). Hence, microwave assisted extraction may be adopted for effective extraction of chitosan from fungal biomass.

The microwave-assisted extraction of chitosan from the shellfish waste have been studied (Alishahi et al., 2011; El Knidri et al., 2016; Mahdy Samar et al., 2013; Sagheer et al., 2009) but microwave assisted extraction from fungal biomass has not been reported so far. In this study, optimization of microwave-assisted extraction of the biopolymer from $R$. oryzae NRRL 1526 biomass, using full factorial experimental design and data analysis, was performed. In recent years there has been a revived interest in bio-production of commodity chemicals, especially dicarboxylic acids, via $R$. oryzae mediated fermentation (Martin-Dominguez, Estevez, Ojembarrena, Santos, \& Ladero, 2018; Meussen, de Graaff, Sanders, \& Weusthuis, 2012). Moreover, the fungus is readily used in the food industry, as well as the known producer of enzymes, such as lipase, protease, and pectinase, due to its generally regarded as safe (GRAS) status (Cantabrana, Perise, \& Hernández, 2015; Ghosh \& Rani Ray, 2011). The simultaneous extraction of chitosan from the residual $R$. oryzae biomass after fermentation can have a profound impact on feasibility of a bio-production process of commodity chemicals. In fact, in the closed loop concept, if the residual biomass can be obtained after recovery of primary bioproduct and used to co-extract another important product, it justifies the carbon-neutral approach of bio-production process.

The work performed here investigates the possibility of adopting microwave assisted extraction as an efficient alternative to conventional autoclave mediated extraction of fungal chitosan. This use of more efficient heating source, afforded by the use of microwave, might be able to improve the chitosan yield from fungal biomass. Moreover, the identification of optimal conditions of microwave heating should be able to reduce the amount of energy consumed for fungal chitosan extraction as well as provide higher chitosan yield due to improved heating efficiency.

\section{Materials and methods}

\subsection{Fungal strain, culture conditions and biomass harvesting for optimization of microwave extraction conditions}

R. oryzae NRRL 1526 was obtained from ARS (Agricultural Research Service, US) culture collection. The inoculum was prepared by growing the fungus on potato dextrose agar (PDA) (Nutri-Bact, Quebec, Canada) plates at $30 \pm 1{ }^{\circ} \mathrm{C}$ for 3 days (Chatterjee et al., 2005). The potato dextrose broth (PDB) culture media (Nutri-Bact, Quebec, Canada) were inoculated with one $1 \mathrm{~cm} \times 1 \mathrm{~cm}$ mycelium covered agar block and incubated at $30 \pm 1{ }^{\circ} \mathrm{C}$ for 2 weeks (Chatterjee et al., 2005; Suntornsuk et al., 2002). The mycelial mat obtained was harvested, rinsed with deionized water, frozen and freeze-dried at $-55^{\circ} \mathrm{C}$ (CoolSafe, SCANVAC) as it was faster than drying at high temperature as well as easier to crumble in a blender and hence, no data about yield after drying was determined.

\subsection{Conventional alkali extraction of chitosan}

The extraction of chitosan was carried out, in duplicates, by a modified method described by Suntornsuk et al. (2002). The freeze-dried mycelia were finely ground with a blender, suspended with $1 \mathrm{~N} \mathrm{NaOH}$ (Fisher Chemicals, New Jersey, USA) solution (1:50; w/v) and autoclaved at $121^{\circ} \mathrm{C}$ for $15 \mathrm{~min}$. The alkali-insoluble fraction was collected after centrifugation at $8000 \times g$ for $15 \mathrm{~min}$, washed with distilled water and recentrifuged until neutral $\mathrm{pH}$ was obtained. The residues obtained after washing was dried and alkali insoluble material (AIM) quantified. AIM was further extracted in $2 \%$ acetic acid (1:40; w/v) (Fisher Chemicals, New Jersey, USA) at $95 \pm 1{ }^{\circ} \mathrm{C}$ for $8 \mathrm{~h}$ and the slurry obtained centrifuged at $8000 \times g$ for $15 \mathrm{~min}$ and the acid-insoluble fraction discarded. The $\mathrm{pH}$ of the supernatant obtained was adjusted to $\mathrm{pH} 10$ with $2 \mathrm{~N} \mathrm{NaOH}$. The solution was centrifuged at $12,000 \times g$ for $15 \mathrm{~min}$. The precipitated chitosan was then washed with distilled water and centrifuged and the step was repeated with 95\% ethanol (1:20; $\mathrm{w} / \mathrm{v})$, followed by acetone $(1: 20 ; \mathrm{w} / \mathrm{v})$. The chitosan so obtained was freeze-dried at $-55^{\circ} \mathrm{C}$ (CoolSafe, SCANVAC).

\subsection{Microwave-assisted extraction experimental design and data analysis}

Statistica software (Version 13.3) was used for the design of experiment as well as analysis of experimental data. Full factorial experimental design, with independent variables power and duration of microwave heating ( 2 factors at 3 levels), was used to identify the optimal conditions for MAE. The microwave energy and duration of heating used for the study ranged from 100 to $500 \mathrm{~W}$ and 10 to $30 \mathrm{~min}$ respectively (with concentration of $\mathrm{NaOH}$ kept constant at $1 \mathrm{~N}$ ). Analysis of variance on the experimental data obtained was performed to determine the effect of power and duration of heating on the dependent variables of AIM yield, chitosan yield and degree of deacetylation. The contour plots obtained following RSM analysis using the regression model given by Eq. (1) was used to determine the optimal conditions of MAE of chitosan from $R$. oryzae biomass.

$\mathrm{y}=\beta_{0}+\Sigma \beta_{\mathrm{i}} \mathrm{X}_{\mathrm{i}}+\Sigma \beta_{\mathrm{ii}} \mathrm{X}_{\mathrm{i}}^{2}+\Sigma \Sigma \beta_{\mathrm{ij}} \mathrm{X}_{\mathrm{ij}}$

where, $\mathrm{y}$ is the predicted response, $\beta_{0}$ is the model intercept; $\beta_{\mathrm{i}}$ and $\beta_{\mathrm{ij}}$ are the coefficients of linear terms; $\beta_{\mathrm{ii}}$ is the coefficient of the quadratic term; $\mathrm{X}_{\mathrm{i}}$ and $\mathrm{Xj}$ are the independent variables.

The freeze-dried mycelia were finely ground with a blender, suspended with $1 \mathrm{~N} \mathrm{NaOH}$ solution $(1: 50 ; \mathrm{w} / \mathrm{v})$ and subjected to the dif- 
ferent microwave conditions (in duplicates), with the aid of Perkin Elmer Multiwave microwave sample preparation system (Anton Paar $\mathrm{GmbH}$, Strasse, Austria). The alkali-insoluble fraction was collected after centrifugation at $8000 \times g$ for $15 \mathrm{~min}$, washed with distilled water and recentrifuged until neutral $\mathrm{pH}$ was obtained. The residues obtained after washing was freeze-dried (CoolSafe, SCANVAC) and alkali insoluble material (AIM) quantified. AIM was further extracted in $2 \%$ acetic acid $(1: 40 ; \mathrm{w} / \mathrm{v})$ at $95^{\circ} \mathrm{C}$ for $8 \mathrm{~h}$ and the slurry obtained centrifuged at $8000 \times g$ for $15 \mathrm{~min}$ and the acid-insoluble fraction discarded. The $\mathrm{pH}$ of the supernatant obtained was adjusted to $\mathrm{pH} 10$ with $2 \mathrm{~N} \mathrm{NaOH}$. The solution was centrifuged at $8000 \times g$ for $15 \mathrm{~min}$. Precipitated chitosan was then washed with distilled water, $95 \%$ ethanol $(1: 20 ; \mathrm{w} / \mathrm{v})$ and acetone $(1: 20 ; \mathrm{w} / \mathrm{v})$, respectively, and freeze-dried at $-55^{\circ} \mathrm{C}$ (CoolSafe, SCANVAC). The schematic of the experiment represented in Fig. 1.

\section{Characterization of fungal chitosan}

The determination of the properties of the fungal chitosan, such as DDA and viscosity average molecular weight, discussed below was performed in duplicates.

\subsection{FT-IR studies and determination of the degree of deacetylation (DDA)}

FTIR spectra were recorded in the middle infrared $\left(4000 \mathrm{~cm}^{-1}-400 \mathrm{~cm}^{-1}\right)$ with a resolution of $4 \mathrm{~cm}^{-1}$ in the absorbance mode for 16 scans at room temperature. The various powdered mycelial chitosan samples were subjected to FTIR analysis, equipped with Nicolet iS50 FT-IR with Smart ${ }^{\mathrm{TM}}$ iTX accessory and OMNIC FT-IR software (ThermoFisher Co., Boston, MA, USA). The FT-IR spectra obtained was compared against commercial crab chitosan of DDA 95\% and molecular weight of 100-300 kDa (ACROS Organics, Geel, Belgium). The DD was calculated by measuring the absorbance ratio of $A_{1655}$ and $A_{3450}$. The amide I band at $1655 \mathrm{~cm}^{-1}$ and the hydroxyl group absorption band at $3450 \mathrm{~cm}^{-1}$ were used as internal reference. The equation, proposed by Czechwska Biskup, Diana, Rokita, Ulanski, and Rosiak (2012), used for determination of the degree of deacetylation is as follows using Eq. (2):

Degree of deacetylation

$(\mathrm{DDA})=100-\left[\left(\left(\mathrm{A}_{1655} / \mathrm{A}_{3450}\right) \times 100\right) / 1.33\right]$

\subsection{Determination of degree of deacetylation by potentiometric titration}

The degree of deacetylation was determined by potentiometric titration, according to the method of Czechowska-Biskup et al. (2004). $0.02 \mathrm{~g}$ of dried fungal chitosan, derived using optimum MAE condition as well as conventional autoclave extraction, was dissolved in $20 \mathrm{ml}$ $0.1 \mathrm{M}$ acetic acid. Titration of this solution with a $0.1 \mathrm{M} \mathrm{NaOH}$ solution was performed and a $\mathrm{pH}$ curve with two inflection points obtained. The first derivative of the $\mathrm{pH}$ curve was determined and plotted to pre- cisely identify the volumes of $0.1 \mathrm{M} \mathrm{NaOH}$ used corresponding to the two inflections points. The degree of deacetylation (DDA) of the chitosan samples was then determined, using the volume information, according to the formula (Czechowska-Biskup et al., 2004):

Degree of deacetylation

$(\mathrm{DDA})=2.03 \times\left(\left(\mathrm{V}_{2}-\mathrm{V}_{1}\right) /\left(m+0.0042\left(\mathrm{~V}_{2}-\mathrm{V}_{1}\right)\right)\right)$

where $m$ is the weight of chitosan sample; $V_{2}, V_{1}$ is the volumes of $0.1 \mathrm{M}$ sodium hydroxide solution corresponding to the deflection points.

\subsection{Molecular weight determination}

Extracted chitosan solutions, conventional, as well as microwave extracted, of varying concentrations ranging from 0.0625 to $0.5 \%$ (w/ v), were prepared in $2 \%$ acetic acid (Fisher Scientific, New Hampshire, USA), stirred for $24 \mathrm{~h}$ at room temperature and filtered through $0.45 \mu \mathrm{m}$ glass filters (Fisher Scientific, New Hampshire, USA) to remove insoluble fractions (Chatterjee et al., 2005; Vaingankar \& Juvekar, 2014). The viscosity of the solutions, in duplicates, was determined at $25^{\circ} \mathrm{C}$ using Fungilab rotational viscometer (Fungilab, Barcelona, Spain). The reduced viscosity $\left(\eta_{\text {red }}\right)$ was calculated using Equations below (4)-(6) and plotted against concentration $(\mathrm{C}, \mathrm{g} / 100 \mathrm{ml})$. The intrinsic viscosity $([\eta])$ was obtained by extrapolating the curve to Y-axis.

Relative viscosity, $\eta_{\text {relative }}=$ viscosity of solution/viscosity of solvent (4)

Specific viscosity, $\eta_{\mathrm{sp}}=\eta_{\text {relative }}-1$

Reduced viscosity, $\eta_{\text {red }}=\eta_{\mathrm{sp}} / \mathrm{C}$

The intrinsic viscosity obtained was then used to calculate the viscosity-average molecular weight $\left(\mathrm{M}_{\mathrm{v}}\right)$ of chitosan from the viscosity-molecular weight equation (Sagheer et al., 2009):

$[\eta]=0.078 * \mathrm{M}_{\mathrm{v}}^{0.76}$

\subsection{Crystallinity index determination using $X$-ray powder diffractometry} $(X R D)$

The XRD measurements on powdered chitosan samples, commercial animal derived chitosan and fungal chitosan obtained via MAE as well as conventional autoclave extraction, were carried out to determine their crystal structure. The diffractogram was obtained using benchtop X-ray diffractometer (AERIS, MalvernPANalytical, Malvern, UK). The diffractometer was equipped with $\mathrm{Cu}$ k $\alpha$ radiation $(\lambda=1.5406 \AA)$ and operated at $40 \mathrm{kV}$ and $8 \mathrm{~mA}$. A continuous scan between $2 \theta$ angles of $5^{\circ}$ of $40^{\circ}$ was carried out with a step size of $0.022^{\circ}$ and a scan time of $46.9 \mathrm{~s}$. The crystallinity index $\left(\mathrm{I}_{\mathrm{CR}}\right)$ was calculated using the following equation (El Knidri et al., 2016; Sagheer et al., 2009):

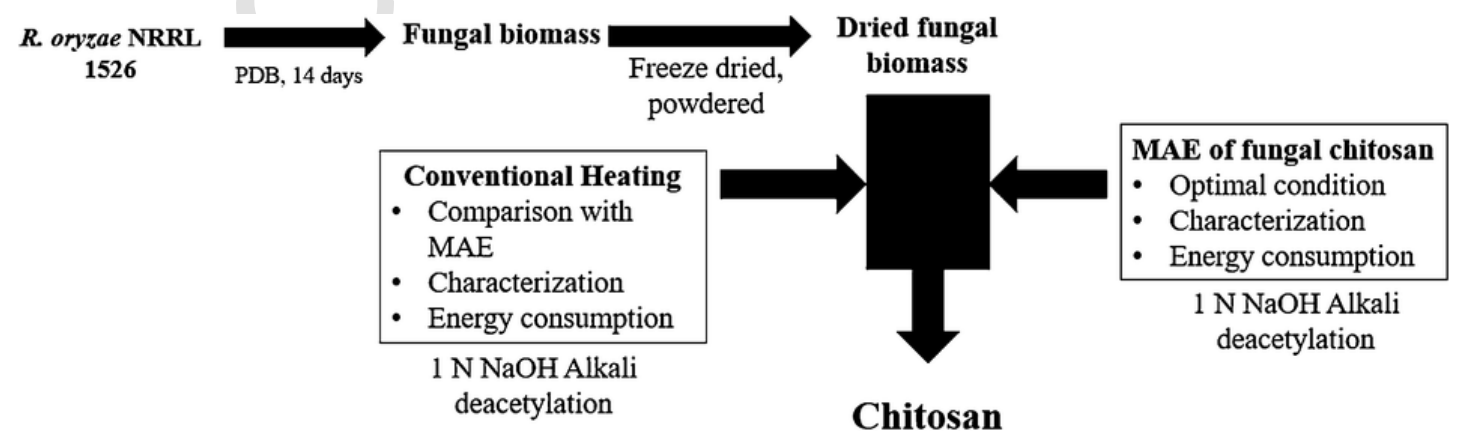

Fig. 1. Schematic of the experiment. 
Crystallinity index $\left(\mathrm{I}_{\mathrm{CR}}\right)=\left(\mathrm{I}_{110}-\mathrm{I}_{\mathrm{am}}\right) / \mathrm{I}_{110} \times 100$

where $I_{a m}$ is the intensity of amorphous diffraction at $2 \theta \cong 16^{\circ}$ and $I_{110}$ is the maximum intensity at $2 \theta \cong 20^{\circ}$.

\section{Energy consumption}

Electrical power consumed during microwave assisted chitosan extraction as well as during conventional method of autoclave extraction from fungal biomass is determined by Eq. (9) (Laadila et al., 2017).

$\mathrm{E}=(\mathrm{P} \times \mathrm{t}) / 1000$

where $\mathrm{E}$ is electric energy consumed during the operation of microwave digester (kWh); $\mathrm{P}$ is power (W) and $\mathrm{t}$ is time (hour).

\section{Results and discussion}

\subsection{Statistical studies and identification of optimal conditions for MAE of} chitosan

The effect of microwave power $\left(\mathrm{X}_{1}\right)$ and time of incubation $\left(\mathrm{X}_{2}\right)$, ranging from $100-500 \mathrm{~W}$ and $10-30 \mathrm{~min}$ respectively, were studied to identify the optimal conditions for maximum chitosan extraction. Full factorial data analysis was performed to obtain an understanding of the effect of each parameter on chitosan extraction and represented in Table 1. The optimum values of independent variables were obtained by applying desirability function (d) to the results of the dependable variables (AIM yield, chitosan yield and degree of deacetylation) given in Table 2 for the best agreement. This was followed by the determination of the global desirability function (D) to determine the optimum condition for microwave assisted chitosan extraction. These results are represented in Fig. 2 and the global desirability illustrated in Fig. 4. The predicted responses are transformed into dimensionless desirability function $\left(\mathrm{d}_{\mathrm{AIM}}\right.$, $\mathrm{d}_{\text {Chitosan, }}$ and $\mathrm{d}_{\mathrm{DDA}}$ ). The objective of this study was to obtain maximum chitosan yield with high DDA and therefore only a single function was used.

$d_{\mathrm{AIM}}=[(\mathrm{AIM}$ yield $-\mathrm{A}) /(\mathrm{B}-\mathrm{A})]$, where $d_{\mathrm{AIM}}=0($ if $\mathrm{AIM}<\mathrm{A})$

and $d_{\mathrm{AIM}}=1$ (if $\mathrm{AIM}>\mathrm{B}$ )

$d_{\text {Chitosan }}=[($ Chitosan yield $-\mathrm{A}) /(\mathrm{B}-\mathrm{A})]$, where $d_{\text {Chitosan }}=0$ (if chitosan yield $<\mathrm{A}$ ) and $d_{\text {Chitosan }}=1$ (if chitosan yield $>$ B)

$d_{\mathrm{DDA}}=[(\mathrm{DDA}-\mathrm{A}) /(\mathrm{B}-\mathrm{A})]$, where $d_{\mathrm{DDA}}=0($ if $\mathrm{DDA}<\mathrm{A})$ and

$d_{\mathrm{DDA}}=1$ (if DDA $>$ B)

where; A and B are the lowest and highest yields obtained for the corresponding response.

The global desirability $(D)$ was obtained by combining the desirability of the individual response, i.e., $d_{\mathrm{AIM}}, d_{\text {Chitosan, }}$ and $d_{\mathrm{DDA}}$ as described by the Equation below:

$D=\left(d_{\text {AIM }} \times d_{\text {Chitosan }} \times d_{\text {DDA }}\right)^{1 / 3}$
When $D>0$, all responses are within the desirable ranges and when $D$ is close to 1 signifies that the combination of the different independent variables (Power and Time) was globally optimum.

The results of ANOVA analysis and interactions are represented in Table 1. Investigation of the effect of the factors, power, and duration of incubation, showed that microwave power had a significant effect on AIM production $(p=0.001)$ whereas duration of microwave treatment had an only slight effect on AIM yield $(p=0.04)$. Fig. 3(a) provides the contour plot that shows the impact of time and microwave power on AIM extraction. Moreover, it was concluded that only power had a linear and quadratic effect on AIM extraction but this effect was not completely independent of the duration of microwave treatment $(p=0.20)$ as can be observed with the p-value for the linear effect. The experimental data have a high degree of correlation with the predicted response $\left(R^{2}=0.982\right)$.

The AIM obtained was then subjected to chitosan extraction. The statistical analysis (ANOVA) of the data showed that microwave power only had an impact on the quantity of chitosan extracted from the AIM $(p=0.01)$. The contour plot of the effect of power and time on chitosan yield is provided in Fig. 3(b) and the experimental data had a lower degree of correlation with predicted response $\left(R^{2}=0.918\right)$. The degree of deacetylation is one of the key parameters that determine the conversion of chitin to chitosan. The effect of different levels of microwave power and duration of the treatment on the degree of deacetylation was investigated. This analysis showed that only power had a significant effect on the degree deacetylation $(p=0.005)$ but the interactive effect of power and time was significant $(p=0.04)$. The experimental data showed a good correlation with the predicted response $\left(R^{2}=0.962\right)$ and the contour plot of the effect of power and time on DDA is represented in Fig. 3(c).

\subsection{Microwave extraction of chitosan and optimization}

Power and duration of microwave treatment were chosen as the variables for the study. Previous studies have used, most commonly, $1 \mathrm{~N}$ $\mathrm{NaOH}$ for the extraction of chitosan from the fungal biomass (Chatterjee et al., 2005; Crestini, Kovac, \& Giovannozzi-Sermanni, 1996; Hu, Hu, Ho, \& Yeung, 2004; Pochanavanich \& Suntornsuk, 2002; Suntornsuk et al., 2002; Tan, 1996; Kleekayai and Suntornsuk, 2010; Vaingankar \& Juvekar, 2014). Hence, this concentration was kept constant during the study and conventional autoclave assisted extraction was used for comparison of the results obtained during microwave extraction. The maximum temperature achieved during different conditions of extraction were $85^{\circ} \mathrm{C}, 200{ }^{\circ} \mathrm{C}, 260^{\circ} \mathrm{C}$ and $121^{\circ} \mathrm{C}$ for $100 \mathrm{~W}, 300 \mathrm{~W}, 500 \mathrm{~W}(30 \mathrm{~min}$ exposure to microwave) and autoclaving, respectively. The quantity and yield of AIM and chitosan obtained during the different extraction conditions and the degree of deacetylation are presented in Table 2. The yields of chitosan and AIM presented in this study are represented with respect to unit biomass dry weight.

The use of least microwave power $(100 \mathrm{~W})$ gave the highest alkali insoluble material (AIM) yield, 23\% (w/w), albeit the lowest chitosan yield of $2.1 \% \mathrm{w} / \mathrm{w}$. This might be due to the low temperature $\left(50^{\circ} \mathrm{C}-85^{\circ} \mathrm{C}\right)$ achieved during the low power condition $(100 \mathrm{~W})$ lead

Table 1

Effect of independent variables ( $\mathrm{X}_{1}$ - Power; $\mathrm{X}_{2}$ - Time) on dependent variables (AIM yield; Chitosan yield; Degree of deacetylation) (Significant values: $p<0.05$ ).

\begin{tabular}{|c|c|c|c|c|c|c|}
\hline \multirow[t]{2}{*}{ Model } & \multicolumn{2}{|c|}{ AIM yield (\% w/w) } & \multicolumn{2}{|c|}{ Chitosan yield (\% w/w) } & \multicolumn{2}{|c|}{ Degree of deacetylation (\%) } \\
\hline & $p$ & Effect & $p$ & Effect & $p$ & Effect \\
\hline $\mathrm{X}_{1}$ & 0.001346 & -20.020 & 0.886201 & 0.3133 & 0.005187 & 6.9400 \\
\hline $\mathrm{X}_{1}^{2}$ & 0.072485 & 4.0367 & 0.012715 & 9.3433 & 0.256050 & 1.1433 \\
\hline $\mathrm{X}_{2}$ & 0.037689 & -6.1067 & 0.209808 & -3.2033 & 0.088072 & 2.3533 \\
\hline $\mathrm{X}_{2}^{2}$ & 0.532541 & -1.0433 & 0.866767 & 0.31833 & 0.339012 & -0.9267 \\
\hline $\mathrm{X}_{1} \mathrm{X}_{2}$ & 0.199119 & -3.4450 & 0.217709 & -3.8350 & 0.036723 & -4.1600 \\
\hline
\end{tabular}


Table 2

AIM yield, chitosan yield and degree of deacetylation.

\begin{tabular}{lllll}
\hline Power $(\mathrm{W})$ & $\begin{array}{l}\text { Time } \\
(\mathrm{min})\end{array}$ & $\begin{array}{l}\text { AIM yield } \\
(\% \mathrm{w} / \mathrm{w})\end{array}$ & $\begin{array}{l}\text { Chitosan yield } \\
(\% \mathrm{w} / \mathrm{w})\end{array}$ & $\begin{array}{l}\text { Degree of } \\
\text { deacetylation }(\%)\end{array}$ \\
\hline \multirow{2}{*}{100} & 10 & $24.63 \pm 3.3$ & $1.69 \pm 0.4$ & $84.31 \pm 0.7$ \\
& 20 & $24.57 \pm 7.8$ & $2.13 \pm 0.4$ & $87.18 \pm 0.3$ \\
300 & 30 & $21.89 \pm 3.1$ & $2.67 \pm 0.7$ & $89.84 \pm 0.1$ \\
& 10 & $20.64 \pm 0.2$ & $12.23 \pm 1.4$ & $90.10 \pm 1.9$ \\
500 & 20 & $17.84 \pm 3.3$ & $14.43 \pm 3.9$ & $90.65 \pm 0.7$ \\
& 30 & $14.69 \pm 2.3$ & $8.33 \pm 0.5$ & $94.42 \pm 1.3$ \\
& 10 & $10.03 \pm 0.1$ & $6.87 \pm 0.5$ & $95.87 \pm 0.9$ \\
Conventional & 20 & $0.60 \pm 4.2$ & $0.38 \pm 0.5$ & $93.20 \pm 0.6$ \\
& 30 & $0.40 \pm 3.5$ & $0.18 \pm 0.1$ & $93.08 \pm 0.5$ \\
& 20 & $22.09 \pm 2.3$ & $6.67 \pm 0.3$ & $90.62 \pm 0.5$ \\
\hline
\end{tabular}

only partial conversion of fungal biomass to chitosan. The AIM primarily might be made up of undigested hydrated fungal biomass. On the other hand, the use of highest microwave power $(500 \mathrm{~W})$ gave the least AIM yield, 3.12\% (w/w), but average chitosan yield, 2.6\% (w/ $\mathrm{w}$ ), was slightly better than that obtained during the lowest power conditions. High temperatures $\left(200^{\circ} \mathrm{C}-260^{\circ} \mathrm{C}\right)$ achieved in this condition might have led to complete digestion of fungal biomass in the presence of $1 \mathrm{~N} \mathrm{NaOH}$. The exact reason for this reduction in both AIM as well as chitosan yield is not clearly understood and might be due to degradation of chitosan. The observed drastic decrease in both AIM and chitosan yield, represented in Table 2, should be able to support this hypothesis. Moreover, the use of microwave has been studied to accelerate the degradation of high molecular weight chitosan to low molecular weight chitosan (Li et al., 2012). Highest chitosan yield was obtained when moderate microwave power condition ( $300 \mathrm{~W}, 20 \mathrm{~min}$ ) was used and the yield under said conditions were higher, $14.43 \pm 3.9 \%(\mathrm{w} / \mathrm{w})$ of dry biomass versus $6.67 \pm 0.3 \%(\mathrm{w} / \mathrm{w})$ of biomass than the conventional autoclave method of chitosan extraction.

The maximum chitosan yield of $14.4 \%(\mathrm{w} / \mathrm{w}$ ) of biomass, under microwave extraction condition, was found to be higher than most of the previous studies that used conventional autoclave assisted chitosan ex-

Power (Watt)

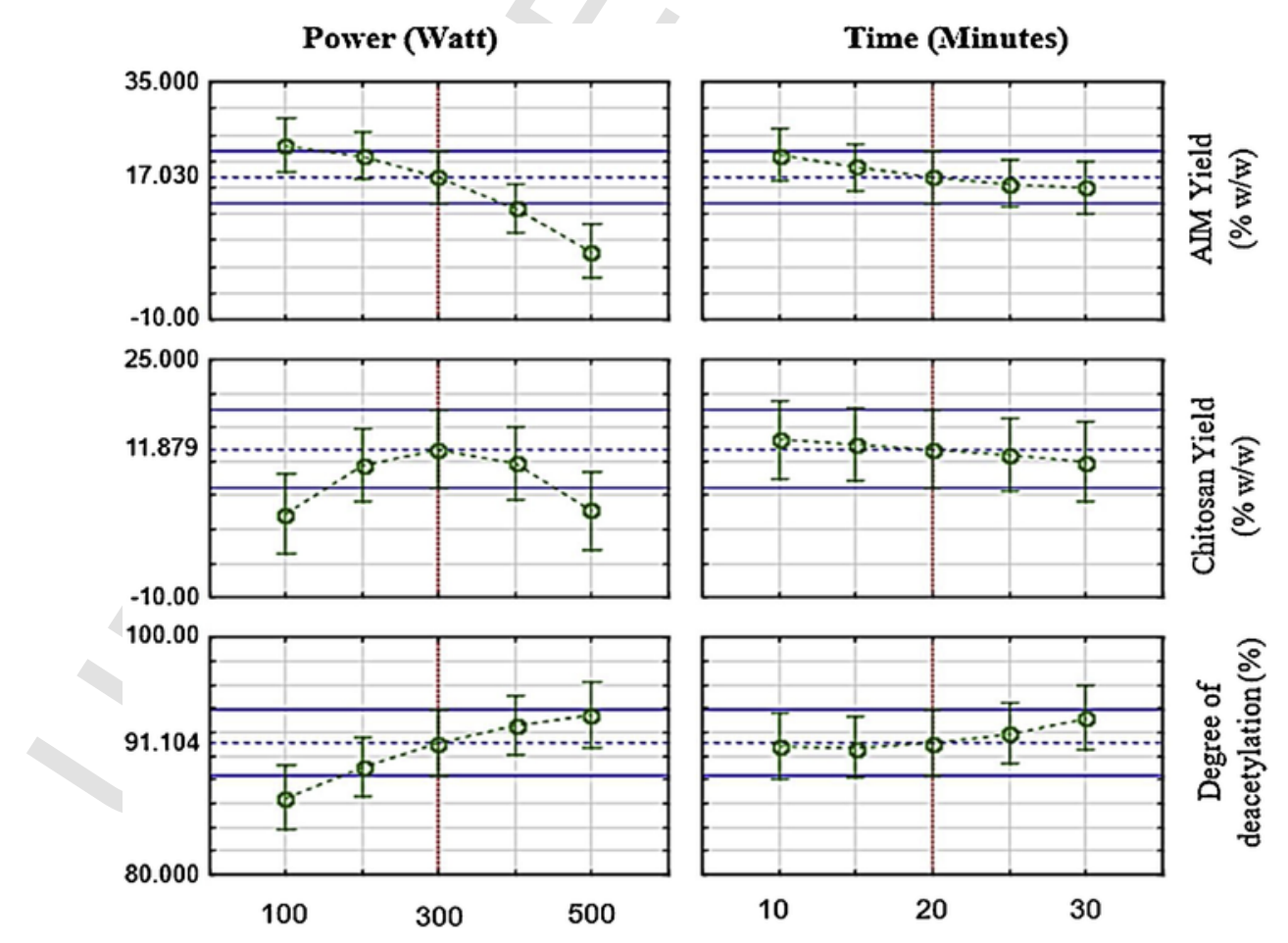

traction. This yield is similar to $14 \%(\mathrm{w} / \mathrm{w})$ reported for $R$. oryzae (Pochanavanich \& Suntornsuk, 2002) and higher than 11.9\% (w/w) obtained from $R$. oryzae grown on deproteinized whey medium (Chatterjee et al., 2008) but lower than the $21 \%(\mathrm{w} / \mathrm{w}$ ) of biomass content reported for Gongronella butleri CCT4274 (Streit et al., 2009). Chitosan yield of $4.3 \mathrm{~g} / \mathrm{kg}$ of the substrate was obtained by culturing $R$. oryzae TISTR3189 on soybean residues during solid-state fermentation (Suntornsuk et al., 2002). Additionally, chitosan yields of up to 406 and $700 \mathrm{mg} / \mathrm{L}$ in corn and rice media, respectively, have been obtained (Hang, 1990). This lack of uniformity in the units, as well as lack of information on total biomass yield or the fermentation media volume, makes it tough to compare chitosan yields with previous studies. The chitosan yield in this study was calculated against biomass obtained. Most of the researchers used $\mathrm{g} / \mathrm{L}$ as the unit for the quantity of chitosan obtained. Since chitosan is extracted from the fungal biomass obtained, it is probably best to express chitosan yield as a function of biomass obtained rather than the amount of media used. The fungal biomass obtained can be expressed as a function of the quantity of media used.

The chitosan yield obtained during this study was found to be higher than those obtained for $R$. arrhizus during the study carried out by Berger et al. (2014). They obtained a maximum yield of $49.31 \mathrm{mg} / \mathrm{g}$. The chitosan yield of $6.67 \%$ using conventional autoclaving technique is in agreement with the $7.1 \%$ obtained for $R$. delemar (Miyoshi, Shimura, Watanabe, \& Onodera, 2014) as well as $6.0-7.7 \%$ obtained for Mucor rouxii (Chatterjee et al., 2005). The maximum chitosan yield, $14.43 \%$ $(\mathrm{w} / \mathrm{w})$, obtained during this study is higher than the $10.7 \%$ and $7.3 \%$ reported for Absidia coerulea 14076 and Mucor rouxii, respectively (Rane \& Hoover, 1993; Synowiecki \& Al-Khateeb, 1997)

AIM yield of $22.09 \%$ obtained by the use of conventional autoclave extraction is in accordance with that reported for $\mathrm{R}$. oryzae (Hu et al., 2004). Hu et al. (2004) screened 33 different strains of fungus for chitosan production. They reported AIM percentage yield of $19.9 \%$ for $R$. oryzae but the chitosan yield obtained was lower than that obtained in this study. This profound difference in chitosan yield, $14.43 \%(\mathrm{w} / \mathrm{w})$ against $0.9 \%(\mathrm{w} / \mathrm{w})$, may be probably due to the difference in the me-

Fig. 2. Predicted profiles of dependent variables time (AIM yield, chitosan yield and degree of deacetylation). 


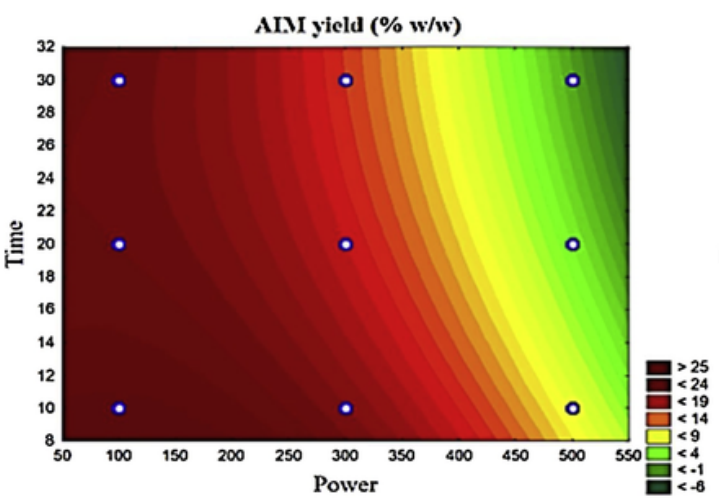

(a)

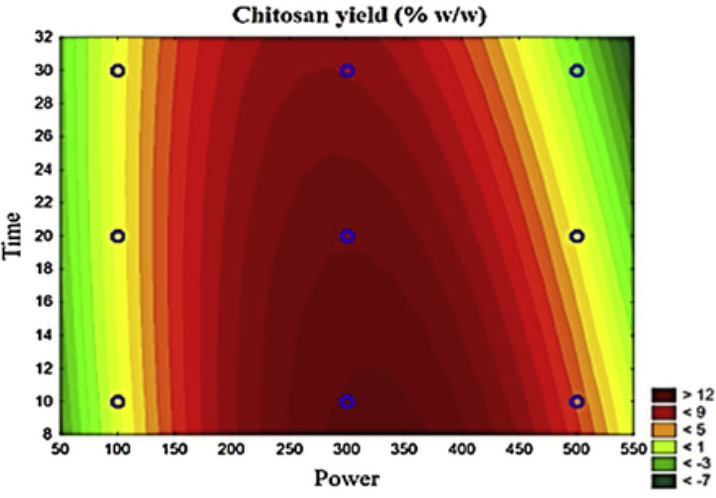

(b)

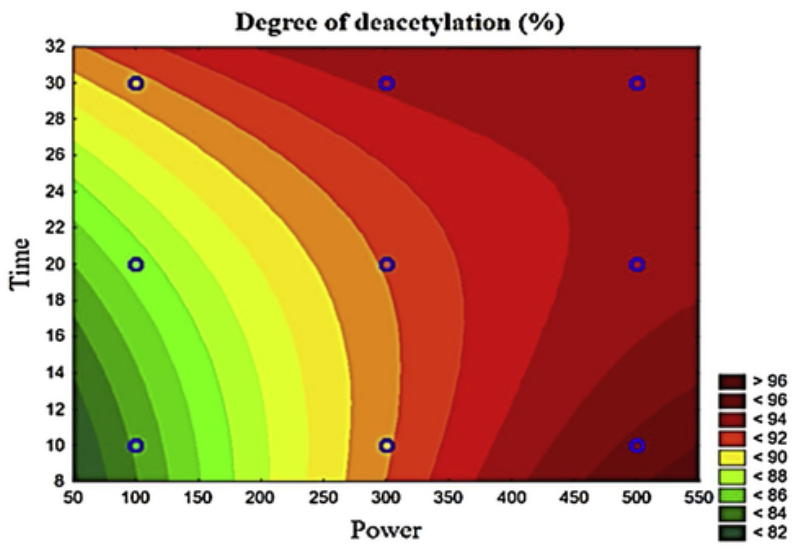

(c)

Fig. 3. Contour plots of the effect of Power (Watt) and Time (minutes) on AIM yield (a), chitosan yield (b) and degree of deacetylation (DDA) (c).

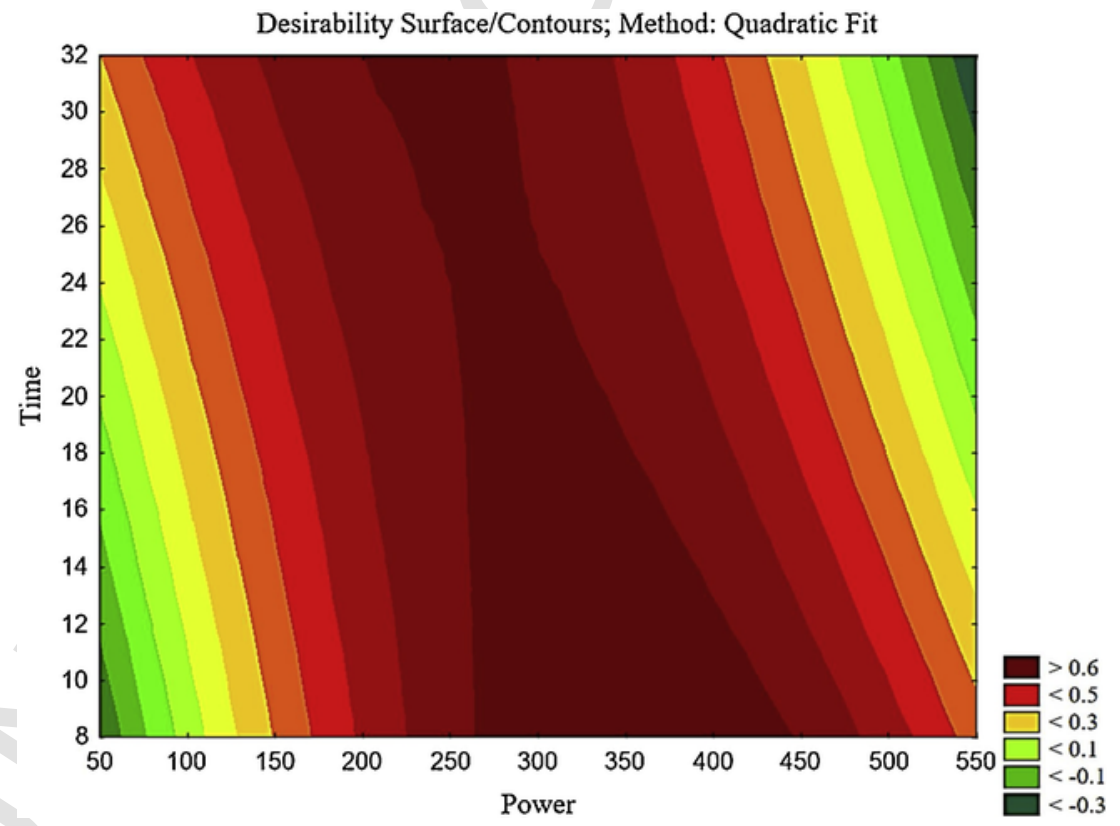

Fig. 4. Global Desirability contour plot.

dia used. PDB was used for this study whereas PGY salt broth, made up of $20(\mathrm{~g} / \mathrm{L})$ glucose, $10(\mathrm{~g} / \mathrm{L})$ peptone, $1(\mathrm{~g} / \mathrm{L})$ yeast extract, $5(\mathrm{~g} / \mathrm{L})$ ammonium sulfate, $1 \mathrm{~g}$ di-potassium hydrogen orthophosphate, $1(\mathrm{~g} / \mathrm{L})$ sodium chloride, $5(\mathrm{~g} / \mathrm{L})$ magnesium sulphate-7-hydrate, $0.1 \mathrm{~g}$ calcium chloride-2-hydrate, was used by $\mathrm{Hu}$ et al. (2004). It has been reported that nitrogen content and type of nitrogen source had an impact on fungal biomass production, chitosan yield and molecular weight of extracted chitosan (Jiang, Pan, \& Kim, 2011). Both temperature and the duration of exposure has been observed to affect chitosan yield from mushrooms (Kannan, Nesakumari, Rajarathinam, \& Ranjit, 2010). 
Hence, the higher temperature of nearly $160{ }^{\circ} \mathrm{C}$ achieved under optimum conditions of microwave extraction probably explains the high yield observed in this study.

Moreover, it was observed that the degree of deacetylation of the chitosan extracted in this study was higher than that obtained by $\mathrm{Hu}$ et al. (2004) for $R$. oryzae. They obtained chitosan of $67.3 \%$ degree to deacetylation whereas chitosan of varying degree of deacetylation ranging from $84 \%$ to $95 \%$ was obtained during this study under various MAE conditions. The degree of deacetylation obtained corresponds to that obtained for $R$. oryzae by Khalaf (2004) as well as similar to that observed by Kleekayai and Suntornsuk (2010) and Pochanavanich and Suntornsuk (2002). The highest degree of deacetylation of $95 \%$ obtained points to the probable effective conversion of chitin to chitosan, in the presence of $\mathrm{NaOH}$, by using higher microwave power.

\subsection{The optimum condition for microwave extraction of chitosan and its characterization}

The theoretical optimal conditions of microwave extraction conditions were $300 \mathrm{~W}$ of microwave power and $22 \mathrm{~min}$, represented in Fig. 3. Fungal biomass was subjected to these optimal conditions of microwave extraction and the chitosan yield obtained evaluated and characterized. This optimal condition of microwave extraction gave a chitosan yield of $13.43 \% \pm 0.3 \%(\mathrm{w} / \mathrm{w})$ and $94.58 \pm 0.9 \%$ degree of deacetylation using FT-IR analysis. The obtained degree of deacetylation was further confirmed by potentiometric titration and was found to be $97.3 \%$ (and $83.9 \%$ for conventional heating) (Supplementary data 2 ). The chitosan yield is similar to the highest chitosan yield represented in Table 2 but was observed to have a higher degree of deacetylation $(94.58 \%$ vs $90.6 \%)$. This increase in the degree of deacetylation rendered by the use of optimal conditions (duration and energy) of microwave heating was statistically confirmed via analysis of variance, which yielded a $p$-value of 0.005 . Moreover, chitosan of higher degree of deacetylation was obtained via microwave heating (optimum conditions) when compared to conventional heating and was found to be significantly higher ( $p$-value $=0.003$ ). Hence, the optimal condition of ( $300 \mathrm{~W}, 22 \mathrm{~min})$ yielded a similar quantity of chitosan but of the higher degree of deacetylation. A similar study that investigated the optimization of microwave extraction conditions for chitosan extractions from Aspergillur niger was performed by Hesheng, Jilong, Chunyuan, and Hongwei (2001). They used ethanol in combination with alkali whereas this study used alkaline treatment alone for deacetylation (Hesheng et al., 2001). Moreover, the optimum condition identified $(480 \mathrm{~W}, 20 \mathrm{~min})$ was higher than the optimum condition presented in Table 3. Additionally, in the previous study, the chitosan obtained was of a lower degree of deacetylation (88\%) compared to that obtained (94\%) in the current study. Additionally, statistical analysis and determination of optimum condition using response surface methodology were performed in the current study and no such study was performed in the previous study. The yields (AIM and chitosan) and properties of chitosan under optimal conditions of MAE are represented in Table 3. The purity of the chitosan obtained was not investigated as defined potato dextrose broth (PDB) was used as the media for culturing $R$. oryzae. Hence, the chances of presence of recalcitrant impurities are minimal. Moreover, the use of high temperature and $1 \mathrm{~N} \mathrm{NaOH}$ for alkaline extraction of chitosan and deacetylation will lead to degradation of proteins as well as sugars.

Microwave-assisted extraction of chitosan from marine crustacean waste has been studied (El Knidri et al., 2016; Sagheer et al., 2009) but not studied for the extraction from fungal biomass. The reduction in the time required to perform deacetylation from hour to minutes was observed in these studies. A similar reduction in the time required for deacetylation was observed wherein the maximum chitosan yield of $14.43 \%$ (w/w) of biomass, with DDA $90.6 \%$, was obtained upon microwave irradiation at $300 \mathrm{~W}$ for only $20 \mathrm{~min}$ whereas chitosan yield of $13.43 \%$, with DDA $94.5 \%$, was obtained under optimum conditions of $300 \mathrm{~W}$ and $22 \mathrm{~min}$. It was observed by Sagheer et al. (2009) that increase in duration of heating lead to an increase in the degree of deacetylation during initial stages of conversion, which plateaus off following a further increase in duration of heating. The rapid increase in temperature achieved via microwave heating due to dielectric heating effect at the molecular level (https://wiki.anton-paar.com/en/ microwave-assisted-synthesis/), most likely helps to achieve a greater degree of deacetylation. Additionally, the reduction in wall effect provided by the use of microwave heating, wherein the walls of the container are heated first followed by gradual increase in the temperature of the reaction medium, leads to a rapid rise in temperature of the reaction medium. This most probably allowed for the effective conversion of chitin to chitosan via removal of the acetyl group from the $\mathrm{N}$-acetylglucosamine monomer moieties that make up chitin.

Moreover, chitosan obtained by MAE $(127.58 \mathrm{kDa})$ was observed to have a comparable molecular weight ( $p$-value 0.58 ) to that obtained via the conventional autoclaving process $(109.08 \mathrm{kDa})$. This, observation is not in accordance with that made by El Knidri et al. (2016) and Sagheer et al. (2009). They observed an increase in molecular weight of chitosan extracted from crustaceans using microwave heating. Sagheer et al. (2009) observed an increase in intrinsic viscosity with duration of microwave heating followed by a decrease in the viscosity. Additionally, microwave assisted heating has also been proposed as a technique for obtaining low molecular weight chitosan (Li et al., 2012).

Table 3

Comparison of chitosan obtained MAE and conventional extraction.

\begin{tabular}{|c|c|c|c|c|c|c|}
\hline \multicolumn{7}{|c|}{ Optimal MAE conditions } \\
\hline Power (W) & Time (min) & AIM yield (\% w/w) & Chitosan yield (\% w/w) & Degree of deacetylation (\%) & & Molecular weight $(\mathrm{kDa})$ \\
\hline $300\left(170^{\circ} \mathrm{C}\right)$ & 22 & $26.11 \pm 4.5$ & $13.43 \pm 0.3$ & $\begin{array}{l}\text { FT-IR calculation } \\
\text { Potentiometric titration }\end{array}$ & $\begin{array}{l}94.58 \pm 0.9^{b} \\
97.28 \pm 0.1^{d}\end{array}$ & $127.58 \pm 33.9^{a}$ \\
\hline \multicolumn{7}{|c|}{ Conventional extraction } \\
\hline Condition & Time & ld $(\%$ w/w) & Chitosan yield (\% w/w) & Degree of deacetylation (\%) & & Molecular weight (kDa) \\
\hline $121^{\circ} \mathrm{C}$ & $20 \square 22$ & 2.3 & $6.67 \pm 0.3$ & $\begin{array}{l}\text { FT-IR calculation } \\
\text { Potentiometric titration }\end{array}$ & $\begin{array}{l}90.62 \pm 0.5^{\mathrm{c}} \\
83.88 \pm 0.1^{\mathrm{e}}\end{array}$ & $109 \pm 20.5^{\mathrm{a}}$ \\
\hline
\end{tabular}

${ }^{\mathrm{i}}$ Each value is the mean of two independent replicate.

${ }^{i i}$ Each value is the mean of two independent replicate.

${ }^{a} \mathrm{p}$-value $>0.05$.

b,c $\mathrm{p}$-value $<0.05$ (DDA calculated from FT-IR data).

${ }^{\mathrm{d}, \mathrm{e}} \mathrm{p}$-value $<0.05$ (DDA calculated using potentiometric titration). 
Hence, the optimal conditions of microwave heating used in this study might not have led to the degradation of the fungal chitosan and yielded chitosan of molecular weight similar to conventional heating. The molecular weights obtained in this study for fungal chitosan, $109 \mathrm{kDA}$ (conventional) and $127 \mathrm{kDA}$ (MAE), is within the range reported for $R$. oryzae, 80-128 kDA, by Kleekayai and Suntornsuk (2010) and similar to $120 \mathrm{kDa}$ reported by Chatterjee et al. (2008).

The X-ray diffraction study of the crystal structure, presented in Fig. 5 , showed that the adoption of MAE did not have a profound impact on the crystal structure of the extracted fungal chitosan. The diffraction pattern of chitosan extracted from $R$. oryzae was similar to commercial animal derived chitosan. Both fungal chitosan extracted by conventional heating and microwave showed the characteristic two distinct peaks corresponding to $2 \theta$ angles of $10^{\circ}$ and $20^{\circ}$ (El Knidri et al., 2016). The calculation of crystallinity index from the diffractogram showed that the crystalline structure of a microwave and conventionally extracted chitosan were similar ( 0.56 for conventional; 0.57 for microwave). This observation is in accordance with that of El Knidri et al. (2016). They observed a similar crystalline structure for the microwave as well as conventional heating extracted chitosan.

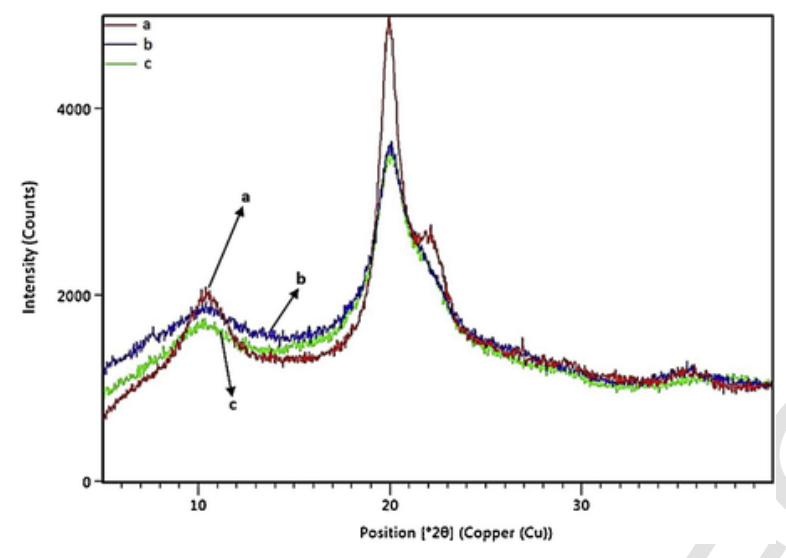

The FT-IR spectrum of chitosan samples along with fungal mycelia is represented in Fig. 6. The FT-IR spectrum of fungal chitosan extracted by microwave irradiation and conventional heating show a high degree of similarity to that of commercial animal derived chitosan. The fungal mycelia show a different pattern compared to chitosan sample, especially around absorbance band $1654 \mathrm{~cm}^{-1}$, which corresponds to amide I. Absorbance corresponding to the bands $3450 \mathrm{~cm}^{-1}$, which corresponds to $\mathrm{OH}$ stretching, and $1655 \mathrm{~cm}^{-1}$ was used to calculate the degree of deacetylation (Czechwska-Biskup et al., 2012; El Knidri et al., 2016). The reduction in the absorbance corresponding to the band $1655 \mathrm{~cm}^{-1}$ shows the effectiveness of the deacetylation process.

Microwave-assisted extraction (MAE) and pressurized liquid extraction (PLE) were compared to obtain polysaccharides, (particularly biologically active $\beta$-glucans) from the fruiting bodies of mushrooms Pleurotus ostreatus and Ganoderma lucidum by Smiderle et al. (2017). They observed that temperature had a stronger influence on the extraction than time (Smiderle et al., 2017). A similar observation was made during this study. It was observed that an increase in power from $100 \mathrm{~W}$ to $300 \mathrm{~W}$, with a corresponding increase in temperature from $85^{\circ} \mathrm{C}$ to $200^{\circ} \mathrm{C}$, led to higher chitosan yield. Microwave irradiation as a tool for DNA extraction has been investigated and these studies have shown that microwave treatment is an efficient and reliable technique for extraction of DNA from fungal biomass for use in PCR (Tendulkar, Gupta, \& Chattoo, 2003; Zhang, Zhang, Liu, Wen, \& Wang, 2010). The suitability of the use of microwave irradiation for biological polysaccharide isolation was further proven by the study performed by $\mathrm{Xu}, \mathrm{Hou}, \mathrm{Hu}$, and $\mathrm{Liu}$ (2018). They optimized the microwave extraction process for isolation of biological polysaccharide from Eucommia ulmoides Oliver leaf. The biological polysaccharide so obtained was characterized and investigated for its antioxidant properties. It was concluded that the optimized microwave extraction condition provided 2.9-fold higher polysaccharide yield than that of the conventional heat reflux extraction method $(\mathrm{Xu}$ et al., 2018). Hence, microwave extraction is a promising technique for the extraction of valuable biomolecules.

Fig. 5. XRD pattern of animal-derived commercial chitosan (a), conventional autoclave assisted extracted fungal chitosan (b) and microwave-assisted extracted chitosan (c).

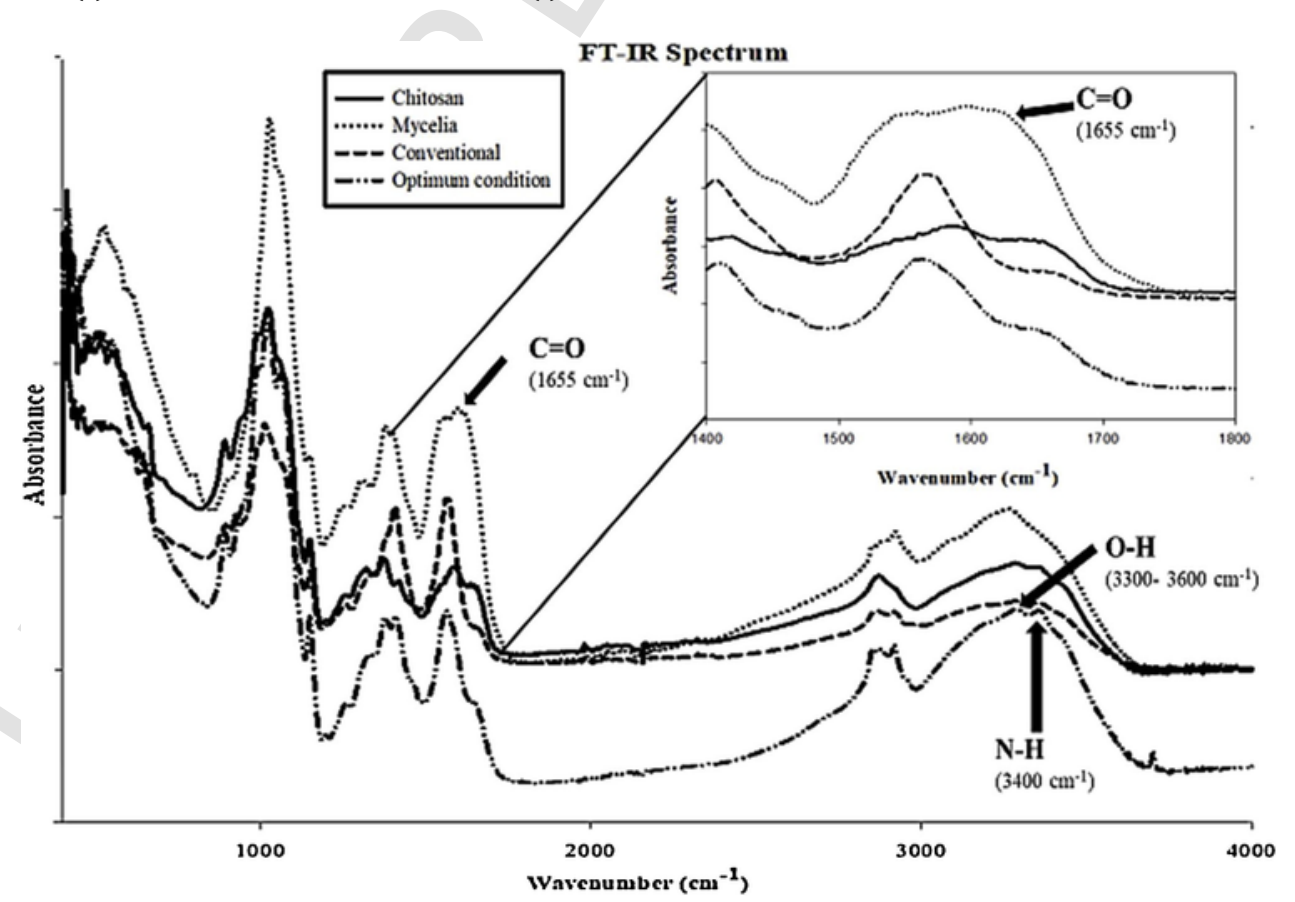

Fig. 6. FT-IR spectrum of commercial crab chitosan, fungal mycelia and chitosan obtained after extraction (conventional and optimum microwave condition identified). 


\subsection{Energy calculations}

The main benefit of adoption of microwave-assisted extraction is the significantly reduced energy consumption. The conventional autoclave method consumes a larger amount of energy to achieve the temperature required for effective deacetylation of fungal chitin and deproteination. For instance, the autoclave used for the study uses a $6000 \mathrm{~W}$ heater that needs to be operated for $50 \mathrm{~min}$ to attain and maintain a temperature of $121^{\circ} \mathrm{C}$. This accounts for energy consumption of $5 \mathrm{kWh}$ and amounts to 50 cents per run (assuming 10 cents/kWh for medium scale classification of electricity supply) (https://www.hydro.mb.ca). On the other hand, the efficient heating achieved by microwave leads to the energy consumption of $0.11 \mathrm{kWh}$ operating under optimal conditions $(300 \mathrm{~W}$, $22 \mathrm{~min}$ ) identified in this study. This energy consumption only amounts to 1.1 cents per run for the microwave digester used for the study. The considerable difference in this operational cost associated with chitosan extraction can have a profound impact on the feasibility of a commercial establishment aimed at fungal chitosan production. Affordable industrial microwave systems are available but they need to be optimized or modified for chitosan extraction prior to commercial production. Hence, MAE of chitosan provides an attractive alternative to conventional fungal chitosan extraction.

\section{Conclusion}

The study here identified the optimal conditions of microwave-assisted extraction (MAE) of chitosan from the biomass obtained by culturing $R$. oryzae NRRL 1526 to be $300 \mathrm{~W}$ and $22 \mathrm{~min}$. The chitosan obtained under the optimal conditions of MAE, compared to conventional heating, showed a higher degree of deacetylation ( $94.6 \%$ against $90.6 \%)$. However, the molecular weight as well as crystalline structure were found to be similar. Additionally, it was observed that MAE of chitosan from fungal biomass yielded a higher amount of chitosan when compared to conventional heating, $13.43 \%$ ( $\approx 2$-fold) versus $6.67 \%$ (w/ w) of fungal biomass. This increase in chitosan yield in addition to the significant reduction in the energy bill, 1.1 cents against 50 cents for conventional heating, can have a profound impact on the feasibility and attractiveness of commercial production of fungal chitosan. The use of microwave-assisted heating in the current study has shown to improve chitosan yield as well as reduce energy consumption when compared to conventional heating. Thus, MAE-optimized extraction of chitosan from fungal biomass can be an attractive and feasible alternative to animal chitosan.

\section{Declarations of interest}

None.

https://www.hydro.mb.ca/accounts_and_services/rates/pdf/surveyof-canadian-electricity-bills.pdf (Accessed 04 August 2018).

https://wiki.anton-paar.com/en/microwave-assisted-synthesis/ (Accessed 10 January 2019).

\section{Acknowledgments}

The authors are sincerely thankful for the financial support of NSERC (Discovery Grants Program) and the Ministère des Relations Internationales du Québec (coopération Paraná-Québec 2010-2012). The views and opinions expressed in this review are those of the authors.

\section{Appendix A. Supplementary data}

Supplementary material related to this article can be found, in the online version, at doi:https://doi.org/10.1016/j.carbpol.2019.05.047.

\section{References}

Alishahi, A., Mirvaghefi, A., Tehrani, M.R., Farahmand, H., Shojaosadati, S.A., Dorkoosh, F.A., Elsabee, M.Z., 2011. Enhancement and characterization of chitosan extraction from the wastes of shrimp packaging plants. Journal of Polymers and the Environment 19 (3), 776-783.

Amorim, R.V.d.S., Souza, W.d., Fukushima, K., Campos-Takaki, G.M.d., 2001. Faster chitosan production by mucoralean strains in submerged culture. Brazilian Journal of Microbiology 32 (1), 20-23.

Berger, L., Stamford, T., Stamford-Arnaud, T., de Alcântara, S., da Silva, A., da Silva, A., .. de Campos-Takaki, G., 2014. Green conversion of agroindustrial wastes into chitin and chitosan by Rhizopus arrhizus and Cunninghamella elegans strains. International Journal of Molecular Sciences 15 (5), 9082-9102.

Brown, D., Brunt, K., Rehmann, N., 2016. Chitosan biopolymer from fungal fermentation for delivery of chemotherapeutic agents. Material Matters 11 (3), 86-89.

Cantabrana, I., Perise, R., Hernández, I., 2015. Uses of Rhizopus oryzae in the kitchen. International Journal of Gastronomy and Food Science 2 (2), 103-111.

Chatterjee, S., Adhya, M., Guha, A.K., Chatterjee, B.P., 2005. Chitosan from Mucor rouxii: Production and physico-chemical characterization. Process Biochemistry 40 (1), 395-400.

Chatterjee, S., Chatterjee, S., Chatterjee, B.P., Guha, A.K., 2008. Enhancement of growth and chitosan production by Rhizopus oryzae in whey medium by plant growth hormones. International Journal of Biological Macromolecules 42 (2), 120-126.

Crestini, C., Kovac, B., Giovannozzi-Sermanni, G., 1996. Production and isolation of chitosan by submerged and solid-state fermentation from Lentinus edodes. Biotechnology and Bioengineering 50 (2), 207-210.

Czechowska-Biskup, R, Ulański, P, Rosiak, JM, Kumor, A, Kozak-Michałowska, I, Lorenc, J, Meler, J, 2004. Examination of plant fat- and cholesterol binding by chitosan of various molecular weights - preliminary data. In: In: Struszczyk, H. (Ed.), Progress on of various molecular weights - preliminary data Vol. X, pp. 121-130, Lodz.

Czechwska-Biskup, R., Diana, J., Rokita, B., Ulanski, P., Rosiak, J.M., 2012. Determination of degree of deacetylation of chitosan comparison of methods. Progress on Chemistry and Application of Chitin and its Derivatives 17, 5-20.

Dhillon, G.S., Kaur, S., Brar, S.K., Verma, M., 2012. Green synthesis approach: Extraction of chitosan from fungus mycelia. Critical Reviews in Biotechnology 33 (4), 379-403.

El Knidri, H., El Khalfaouy, R., Laajeb, A., Addaou, A., Lahsini, A., 2016. Eco-friendly extraction and characterization of chitin and chitosan from the shrimp shell waste via microwave irradiation. Process Safety and Environmental Protection 104, 395-405.

Ghosh, B., Rani Ray, R., 2011. Current commercial perspective of Rhizopus oryzae: A review. Journal of Applied Sciences 11 (14), 2470-2486

Hang, Y.D., 1990. Chitosan production from Rhizopus oryzae mycelia. Biotechnology Letters 12 (12), 911-912.

Hesheng, Y., Jilong, W., Chunyuan, Z., Hongwei, G., 2001. The study of microwave technique used in extracting chitosan from Aspergillus niger. Petrochemical Technology 8 (4), 222-224.

Hu, K.-J., Hu, J.-L., Ho, K.-P., Yeung, K.-W., 2004. Screening of fungi for chitosan producers, and copper adsorption capacity of fungal chitosan and chitosanaceous materials. Carbohydrate Polymers 58 (1), 45-52.

Jiang, L., Pan, S., Kim, J.M., 2011. Influence of nitrogen source on chitosan production carried out by Absidia coerulea CTCC AF 93105. Carbohydrate Polymers 86 (1), 359-361.

Kannan, M., Nesakumari, M., Rajarathinam, K., Ranjit, S.A.J.A, 2010. Production and characterization of mushroom chitosan under solid-state fermentation conditions. Advances in Biological Research 4, 10-13.

Kleekayai, T., Suntornsuk, W., 2010. Production and characterization of chitosan obtained from Rhizopus oryzae grown on potato chip processing waste. World Journal of Microbiology and Biotechnology 27 (5), 1145-1154.

Knorr, D., Klein, J., 1986. Production and conversion of chitosan with cultures of Mucor rouxii or Phycomyces blakesleeanus. Biotechnology Letters 8 (10), 691-694.

Kumari, S., Rath, P.K., 2014. Extraction and characterization of chitin and chitosan from (Labeo rohit) fish scales. Procedia Materials Science 6, 482-489.

Laadila, M.A., Hegde, K., Rouissi, T., Brar, S.K., Galvez, R., Sorelli, L., ... Abokitse, K., 2017. Green synthesis of novel biocomposites from treated cellulosic fibers and recycled bio-plastic polylactic acid. Journal of Cleaner Production 164, 575-586.

Li, K., Xing, R., Liu, S., Qin, Y., Meng, X., Li, P., 2012. Microwave-assisted degradation of chitosan for a possible use in inhibiting crop pathogenic fungi. International Journal of Biological Macromolecules 51 (5), 767-773.

Mahdy Samar, M., El-Kalyoubi, M.H., Khalaf, M.M., Abd El-Razik, M.M., 2013. Physicochemical, functional, antioxidant and antibacterial properties of chitosan extracted from shrimp wastes by microwave technique. Annals of Agricultural Sciences 58 (1), 33-41.

Martin-Dominguez, V., Estevez, J., Ojembarrena, F., Santos, V., Ladero, M., 2018. Fumaric acid production: A biorefinery perspective. Fermentation 4 (2).

Meussen, B.J., de Graaff, L.H., Sanders, J.P.M., Weusthuis, R.A., 2012. Metabolic engineering of Rhizopus oryzae for the production of platform chemicals. Applied Microbiology and Biotechnology 94 (4), 875-886. 
Miyoshi, H., Shimura, K., Watanabe, K., Onodera, K., 2014. Characterization of some fungal chitosans. Bioscience, Biotechnology, and Biochemistry 56 (12), 1901-1905.

Mourya, V.K., Inamdar, N.N., 2008. Chitosan-modifications and applications: Opportunities galore. Reactive and Functional Polymers 68 (6), 1013-1051.

Muzzarelli, R., Ilari, P., Tarsi, R., Dubini, B., Xia, W., 1994. Chitosan from Absidia coerulea. Carbohydrate Polymers 25 (1), 45-50.

Nwe, N., Stevens, W.F., 2004. Effect of urea on fungal chitosan production in solid substrate fermentation. Process Biochemistry 39 (11), 1639-1642.

Pochanavanich, P., Suntornsuk, W., 2002. Fungal chitosan production and its characterization. Letters in Applied Microbiology 35 (1), 17-21.

Rajeshkumar, S., Venkatesan, C., Sarathi, M., Sarathbabu, V., Thomas, J., Anver Basha, K., Sahul Hameed, A.S., 2009. Oral delivery of DNA construct using chitosan nanoparticles to protect the shrimp from white spot syndrome virus (WSSV). Fish \& Shellfish Immunology 26 (3), 429-437.

Rane, K.D., Hoover, D.G., 1993. An evaluation of alkali and acid treatments for chitosan extraction from fungi. Process Biochemistry 28 (2), 115-118.

Rinaudo, M., 2006. Chitin and chitosan: Properties and applications. Progress in Polymer Science 31 (7), 603-632.

Sagheer, F.A.A., Al-Sughayer, M.A., Muslim, S., Elsabee, M.Z., 2009. Extraction and characterization of chitin and chitosan from marine sources in Arabian Gulf. Carbohydrate Polymers 77 (2), 410-419.

Smiderle, F.R., Morales, D., Gil-Ramírez, A., de Jesus, L.I., Gilbert-López, B., Iacomini, M., Soler-Rivas, C., 2017. Evaluation of microwave-assisted and pressurized liquid extractions to obtain $\beta$-D-glucans from mushrooms. Carbohydrate Polymers 156, 165-174.
Streit, F., Koch, F., Laranjeira, M.C.M., Ninow, J.L., 2009. Production of fungal chitosan in liquid cultivation using apple pomace as substrate. Brazilian Journal of Microbiology 40 (1), 20-25.

Suntornsuk, W., Pochanavanich, P., Suntornsuk, L., 2002. Fungal chitosan production on food processing by-products. Process Biochemistry 37 (7), 727-729.

Synowiecki, J., Al-Khateeb, N.A.A.Q., 1997. Mycelia of Mucor rouxii as a source of chitin and chitosan. Food Chemistry 60 (4), 605-610.

Tan, S., 1996. The chitosan yield of zygomycetes at their optimum harvesting time. Carbohydrate Polymers 30 (4), 239-242.

Tendulkar, S.R., Gupta, A., Chattoo, B.B., 2003. A simple protocol for isolation of fungal DNA. Biotechnology Letters 25 (22), 1941-1944.

Vaingankar, P.N., Juvekar, A.R., 2014. Fermentative production of mycelial chitosan from zygomycetes: Media optimization and physico-chemical characterization. Advances in Bioscience and Biotechnology 05 (12), 940-956.

White, S.A., Farina, P.R., Fulton, I., 1979. Production and isolation of chitosan from Mucor rouxii. Applied and Environmental Microbiology 38 (2), 323-328.

Xu, J., Hou, H., Hu, J., Liu, B., 2018. Optimized microwave extraction, characterization and antioxidant capacity of biological polysaccharides from Eucommia ulmoides Oliver leaf. Scientific Reports 8 (1), 1-10.

Yokoi, H., Aratake, T., Nishio, S., Hirose, J., Hayashi, S., Takasaki, Y., 1998. Chitosan production from Shochu distillery wastewater by funguses. Journal of Fermentation and Bioengineering 85 (2), 246-249.

Zhang, Y.J., Zhang, S., Liu, X.Z., Wen, H.A., Wang, M., 2010. A simple method of genomic DNA extraction suitable for analysis of bulk fungal strains. Letters in Applied Microbiology 51, 114-118. 